\title{
PHYSICAL PARAMETERS OF THE OLD OPEN CLUSTER TRUMPLER 5
}

\author{
Sang Chul Kim ${ }^{1}$ and Hwankyung Sung ${ }^{2}$ \\ 1 Korea Astronomy Observatory, Taejon 305-348, Republic of Korea \\ 2 Sejong University, Seoul 143-747, Republic of Korea \\ E-mail: sckim@kao.re.kr, sungh@arcsec.sejong.ac.kr \\ (Received Mar. 11, 2003; Accepted Mar. 17, 2003)
}

\begin{abstract}
We present a study of the old open cluster Trumpler 5 ( $\operatorname{Tr} 5$ ), based on the CDS archival data. From the color-magnitude diagrams of $\operatorname{Tr} 5$, we have found the positions of main-sequence turn-off (MSTO) and red giant clump (RGC) stars. Using the mean magnitude of the RGC stars, we have estimated the reddening toward $\operatorname{Tr} 5, E(B-V)=0.60 \pm 0.10$. Using the stars common in two data sets and the theoretical isochrones of Padova group, we have estimated the distance modulus $V_{0}-M_{V}=12.64 \pm 0.20(\mathrm{~d}=3.4 \pm 0.3 \mathrm{kpc})$, the metallicity $[\mathrm{Fe} / \mathrm{H}]=-0.30 \pm 0.10$, and the age of $2.4 \pm 0.2 \mathrm{Gyr}(\log t=9.38$ ). These metallicity and distance values are consistent with the relation between the metallicity and the Galactocentric distance of other old open clusters, for which we obtain the slope of $\Delta[\mathrm{Fe} / \mathrm{H}] / R_{g c}=-0.064 \pm 0.010 \mathrm{dex} \mathrm{kpc}-1$.
\end{abstract}

Key words : open clusters and associations: individual (Trumpler 5) - Galaxy; disk - Galaxy: stellar content - Galaxy: structure - Hertzsprung-Russell diagram

\section{INTRODUCTION}

Old open clusters, with ages greater than $\sim 1$ Gyr, are an important tool for the study of the formation and the early evolution of the Galactic disk (Friel 1995; Chen, Hou, \& Wang 2003). Trumpler $5\left(\operatorname{Tr} 5 ; \alpha=06^{h}\right.$ $36^{m} 32^{s}, \delta=+09^{\circ} 29^{\prime} 25^{\prime \prime}$, J2000.0) is located in antiGalactic center region with very small galactic latitude $\left(l=202 .^{\circ} 86, b=+01 .^{\circ} 05\right)$.

Dow \& Hawarden (1970) obtained distance modulus $(m-M)_{V}=14.40$ and reddening $E(B-V)=0.80$ which give a true distance modulus of $(m-M)_{0}=11.92$ $(\mathrm{d}=2.4 \mathrm{kpc})$ using total-to-selective extinction ratio of $R=3.1$, though their color-magnitude diagram have some scatter (Janes \& Adler 1982). Kalinowski (1974) and Kalinowski et al. (1974) observed a very red $(B-V \approx 6.1 \mathrm{mag}$ ) carbon star, V493 Mon; and suggested that this star is possibly a member of $\operatorname{Tr} 5$ adopting $(m-M)_{0}=12.3 \pm 0.1(\mathrm{~d}=2.9 \pm 0.1 \mathrm{kpc})$ and $E(B-V)=0.48$ for $\operatorname{Tr} 5$. Kalinowski (1975) performed photoelectric and photographic photometry for 1300 stars in $\operatorname{Tr} 5$, and presented the visual absorption $A_{V} \approx 2.1 \mathrm{mag}$ and diameter of $\gtrsim 12^{\prime}(8.4$ pc) for $\operatorname{Tr} 5$ at an assumed true distance modulus of $(m-M)_{0}=11.9 \pm 0.3(\mathrm{~d}=2.4 \pm 0.3 \mathrm{kpc})$. Piccirillo, Kalinowski, \& Wing (1977) found three M giants, three $\mathrm{K}$ giants, and another carbon star with a color temperature of about $4000 \mathrm{~K}$. These authors adopted $(m-M)_{0}=11.4(\mathrm{~d}=1.9 \mathrm{kpc})$ and $E(B-V)=0.6$ for $\operatorname{Tr} 5$. Kalinowski (1979) have used $(m-M)_{V}=12.00$ and $E(B-V)=0.64$, which gives $(m-M)_{0}=10.02$ (d=1.0 kpc) using $R=3.1$ (Janes \& Adler 1982). Re-

Corresponding Author: S. C. Kim cently, Kaluzny (1998) have obtained $B V I_{C}$ CCD photometry of $\operatorname{Tr} 5$ using Kitt Peak National Observatory $2.1 \mathrm{~m}$ and $0.9 \mathrm{~m}$ telescopes. He has obtained the parameters for $\operatorname{Tr} 5$ as $(m-M)_{0}=12.4(\mathrm{~d}=3.0 \mathrm{kpc})$, $E(B-V)=0.58$, and age of $4.1 \mathrm{Gyr}(\log t=9.61)$. However, Kaluzny obtained the reddening and age of Tr 5 by comparing the photometric values of $\operatorname{Tr} 5$ and M67 (Montgomery et al. 1993).

In this paper we present a new analysis of the data for $\operatorname{Tr} 5$ obtained by Kaluzny (1998). Section II describes the data sets used in this study and Section III presents the color-magnitude diagrams of Tr 5 . In Sections IV and $V$, the physical parameters of $\operatorname{Tr} 5$, such as reddening, distance, metallicity and age, are derived. Section VI discusses the comparison of the derived results with those of Kaluzny (1998) and the metallicityGalactocentric radius relation. Finally, a summary and conclusions are given in Secion VII.

\section{OBSERVATIONAL MATERIAL}

J. Kaluzny performed the observations of $\operatorname{Tr} 5$ at the Kitt Peak National Observatory on 1990 Nov. 11 (run \#1; KPNO 2.1m telescope + TEK1 CCD), on 1991 Oct. 6 (run \#2; KPNO 2.1m telescope + T1KA CCD), and on 1991 Oct. 10 - 11 (run \# 3; KPNO $0.9 \mathrm{~m}$ telescope + T1KA CCD). The details on the observations and data reduction can be found in Kaluzny (1998). Figure 1 shows the fields covered by the three data sets superposed on the Digitized Sky Survey image of $\operatorname{Tr} 5$. The non-rectangular shape of the field \#3 shows that the photometry data of the field \#3 in CDS does not contain all the data of the stars in the rectangular field.

The data sets from the three observing runs were 
calibrated independently, giving systematic differences less than $0.02 \mathrm{mag}$. The photometric errors in the magnitude from the CDS data given by Kaluzny (1998) are plotted against the $V$ magnitude in Figure 2.

\section{COLOR-MAGNITUDE DIAGRAMS OF TR 5}

Figure 3 shows the $V-(V-I)$ and $V-(B-V)$ colormagnitude diagrams (CMDs) of the measured stars in Tr 5. Upper panels are for the stars of good photometric qualities and lower panels are for all the observed stars. Panels (a) and (d) are for observing run \#1, (b) and (e) are for the run \#2, and (c) and (f) are for the run \#3.

The distinguishable features seen in the CMDs of Figure 3 are: (i) there is a well-defined main-sequence, the top of which is probably located at $(B-V) \approx 1.1$, $(V-I) \approx 1.35$, and $V \approx 16.7 \mathrm{mag}$ (see Figure 2 of Kaluzny (1994) for the definitions of main-sequence turn-off (MSTO)); (ii) there is a well-developed red giant branch and red giant clump (RGC) stars at $(B-$ $V)_{R G C}=1.55,(V-I)_{R G C}=1.82$, and $V_{R G C}=15.10$ $\mathrm{mag}$, which is noted by crosses in the figures; (iii) as it goes from panel (a) to (b) and to (c), the total number of stars increases, the thickness of the main-sequence stars increases and there exist more stars (probably field stars) brighter than the MSTO at $V \sim 16.7 \mathrm{mag}$, which is reasonable and expectable since the observing run $\# 1$ covers the smallest area and the run $\# 3$ covers the widest area.

\section{REDDENING}

Since $\operatorname{Tr} 5$ is located at low Galactic latitude $(b=$ $\left.+01 .{ }^{\circ} 05\right)$, it is expectable that the interstellar reddening toward $\operatorname{Tr} 5$ could be significant. We have estimated the reddening toward $\operatorname{Tr} 5$ using the mean color of the RGC stars. Janes \& Phelps (1994) estimated the mean color and magnitude of the RGC in old open clusters to be $(B-V)_{0, R G C}=0.95 \pm 0.10$, and $M_{V, R G C}=$ $0.90 \pm 0.40$, when the $V$ magnitude difference between the RGC and the main-sequence turn-off of the clusters, $\delta V$, is greater than one. The mean colors of the RGC are estimated to be $(B-V)_{R G C}=1.55 \pm 0.01$ and $(V-I)_{R G C}=1.82 \pm 0.01$, and the corresponding mean magnitude is $V_{R G C}=15.10 \pm 0.05 \mathrm{mag}$. $\delta \mathrm{V}$ is estimated to be $1.6 \pm 0.2 \mathrm{mag}$, and the resulting reddening value is estimated to be $E(B-V)=0.60 \pm 0.10$.

\section{DISTANCE, METALLICITY AND AGE}

Using the mean magnitude of the RGC (Janes \& Phelps 1994) and that of the RGC of $\operatorname{Tr} 5$ obtained in the previous section, we have estimated the distance modulus of $\operatorname{Tr} 5$ as $V-M_{V}=14.2 \pm 0.1$.

For the stars common in the data sets of $\# 1$ and \# 2, we have plotted the color-magnitude diagrams in Figure 4 and superimposed the ZAMS relation (Sung \&

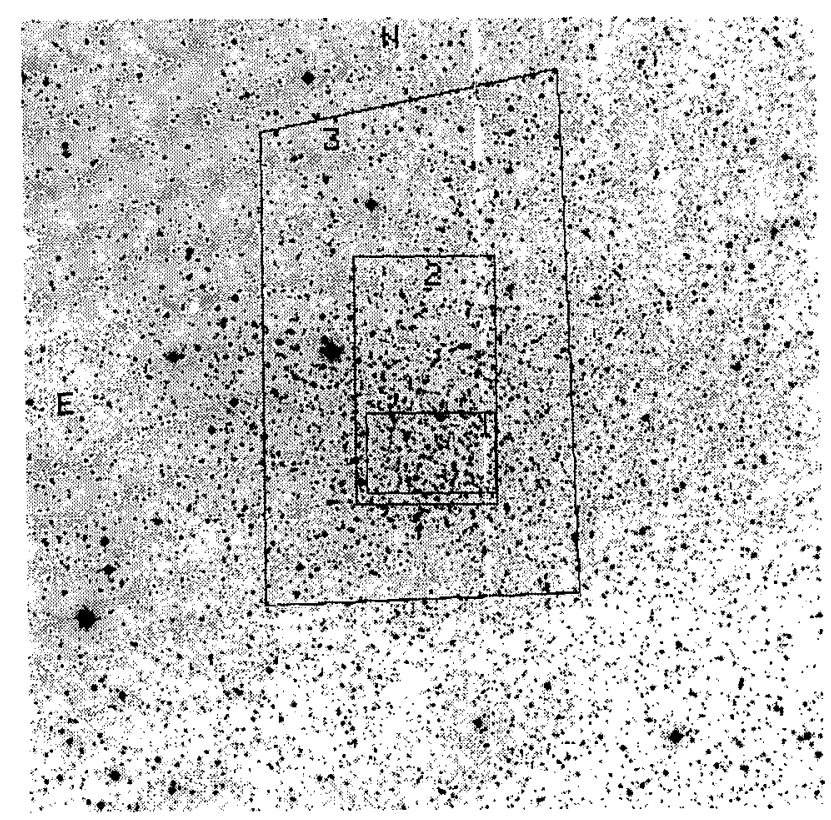

Fig. 1.- The fields covered by the three observing runs superposed on the Digitized Sky Survey image of $\operatorname{Tr} 5$. The center of the image is $\alpha_{2000}=06^{h} 36^{m} 32^{s}$, $\delta_{2000}=+09^{\circ} 29^{\prime} 25^{\prime \prime}$, and the size of the field is $30^{\prime} \times 30^{\prime}$. North is at the top and east is to the left. Regions labeled "1", "2", and "3" represent, respectively, the regions covered by the observing runs of $\# 1, \# 2$, and $\# 3$. See text for the details.

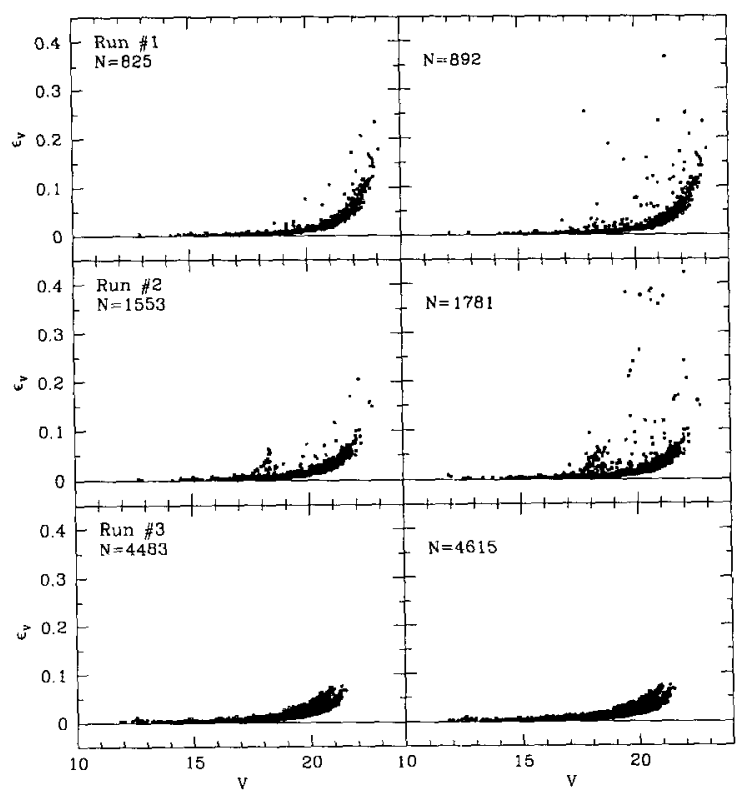

Fig. 2.-- Photometric errors from CDS data given by Kaluzny (1998) as a function of magnitude. Top rows are for observing run \#1, middle rows are for the run \#2, and bottom rows are for the run \#3. Left panels are for stars of good photometric qualities and right panels are for all the observed stars. 


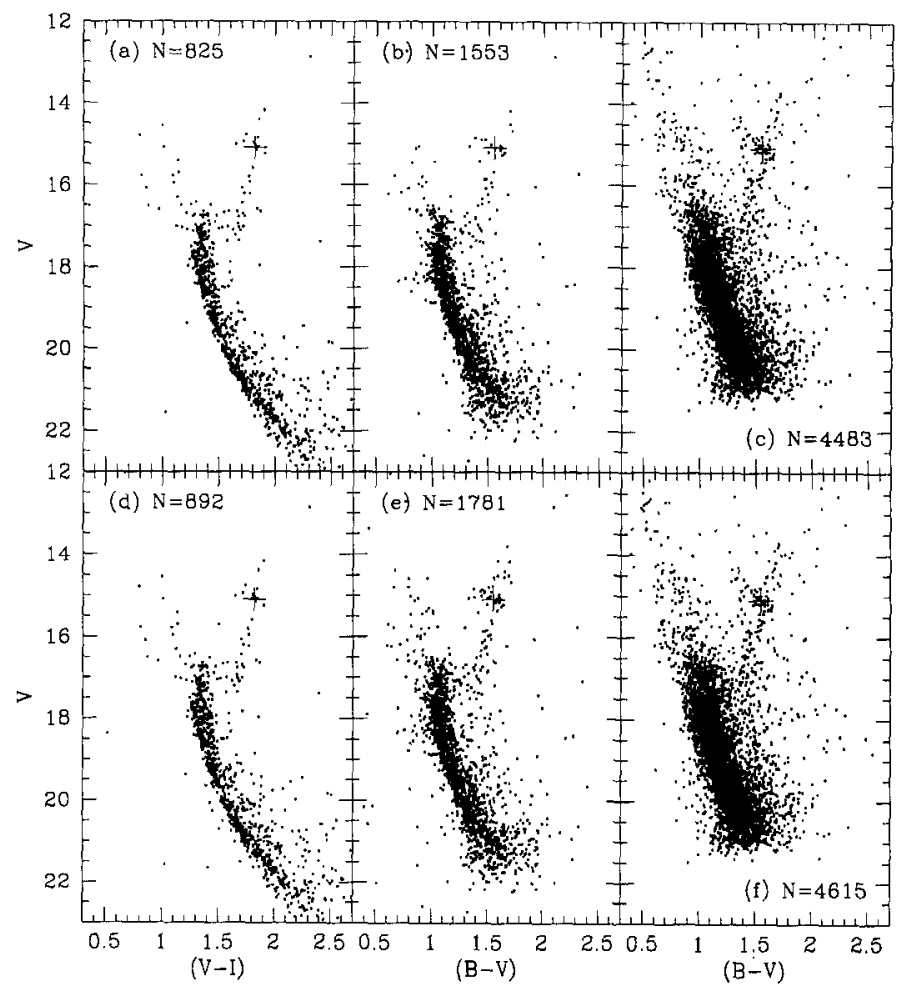

Fig. 3.- Color-magnitude diagrams of Tr 5. Upper panels are for stars of good photometric qualities and lower panels are for all the observed stars. Panesl (a) and (d) are for observing run \#1, (b) and (e) are for the run \#2, and (c) and (f) are for the run \#3. The crosses represent the positions of the red giant clump (RGC).

Bessell 1999; Sung 2001) and the theoretical isochrones of Padova group (Bertelli et al. 1994) which are reddened by $E(B-V)=0.60$ and $E(V-I)=0.80$ and shifted according to the apparent distance modulus of $V-M_{V}=14.6$ (panels (a) and (b)) and $V-M_{V}=14.2$ (panels (c) and (d)). We have used the metallicity of $[\mathrm{Fe} / \mathrm{H}]=-0.30$ and the age of $2.4 \mathrm{Gyr}$ for $\operatorname{Tr}$ 5. The distance modulus of $V-M_{V}=14.6$ gives better fit to the main-sequence and sub-giant branch than that of $V-M_{V}=14.2$. Therefore, we take $V-M_{V}=14.6 \pm 0.2$ as our final value. Using the reddening values of $E(B-V)=0.60$ and $E(V-I)=0.80$ obtained above and the total-to-selective extiontion ratio (Guetter \& Vrba 1989),

$$
R_{V}=\frac{A_{V}}{E(B-V)}=2.45 \frac{E(V-I)}{E(B-V)},
$$

we get $R_{V}=3.26, A_{V}=1.96 \mathrm{mag}$, and the true distance modulus of $V_{0}-M_{V}=12.64 \pm 0.2 \mathrm{mag}$ (d $=3.4 \pm 0.3 \mathrm{kpc}$ ).

We also have estimated the cluster age using the morphological age index (MAI) which was introduced by Phelps, Janes, \& Montgomery (1994). We used the relation between the MAI and $\delta V$ given by Phelps et al. (1994) and Janes \& Phelps (1994),

$$
\operatorname{MAI}[\mathrm{Gyr}]=0.73 \times 10^{\left(0.256 \delta V+0.0662 \delta \mathrm{V}^{2}\right)},
$$

where $\delta V$ represents the $V$ magnitude difference between the RGC and the MSTO of the clusters. From the value of $\delta V$ derived in Section IV, $1.6 \pm 0.2 \mathrm{mag}$, we obtain a value for the age, MAI $=2.8 \pm 0.6 \mathrm{Gyr}$. Even though the MAI was only intended to provide a relative age ranking of clusters, this age is consistent with the value obtained above from the isochrone fitting within the error range. Figure 5 shows the Padova isochrone fitting with three age values of 2.0 (left dotted lines), 2.4 (solid lines), and 2.8 (right dotted lines) Gyr. Finally, we take the age and the metallicity of $\operatorname{Tr} 5$ as $2.4 \pm 0.2 \mathrm{Gyr}$ and $[\mathrm{Fe} / \mathrm{H}]=-0.30 \pm 0.10 \mathrm{dex}$, respectively.

\section{DISCUSSION}

\section{(a) Comparison with the Results of Kaluzny}

Kaluzny (1998) has obtained the age of $\operatorname{Tr} 5$ to be $\sim 4.1$ Gyr by estimating the $\Delta(B-V)$ and $\Delta(V-I)$ (the difference in color between the cluster's MSTO and the red giant branch at the level of the clump). But the difficulty in estimating the position of the MSTO, as he stated, as well as the locus of red giant branch and the position of the RGC itself, gives difference in the obtained ages between that of Kaluzny and ours $(2.4 \mathrm{Gyr})$. Kaluzny also obtained the reddening values of $\operatorname{Tr} 5, E(B-V)=0.58$ and $E(V-I)=0.765$, 

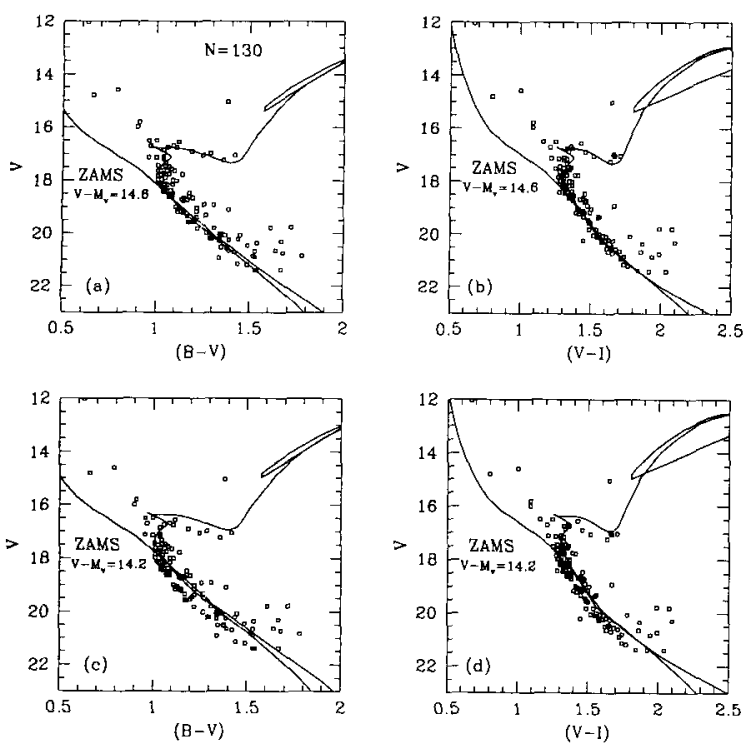

Fig. 4. $-V-(B-V)$ (panels (a) and (c)) and $V-$ $(V-I)$ (panels (b) and (d)) color-magnitude diagrams for the 130 stars commonly contained in the photometry files of observing runs \#1 and \#2. The solid lines represent the ZAMS relation (Sung \& Bessell 1999, Sung 2001) and the Padova isochrones (Bertelli et al. 1994), which are reddened by $E(B-V)=0.60$ and $E(V-I)=0.80$ and shifted according to the distance modulus of $V-M_{V}=14.6$ ((a) and (b)) and $V-M_{V}=14.2((\mathrm{c})$ and (d)). The distance modulus of $V-M_{V}=14.6$ gives better fit to the mainsequence and sub-giant branch.
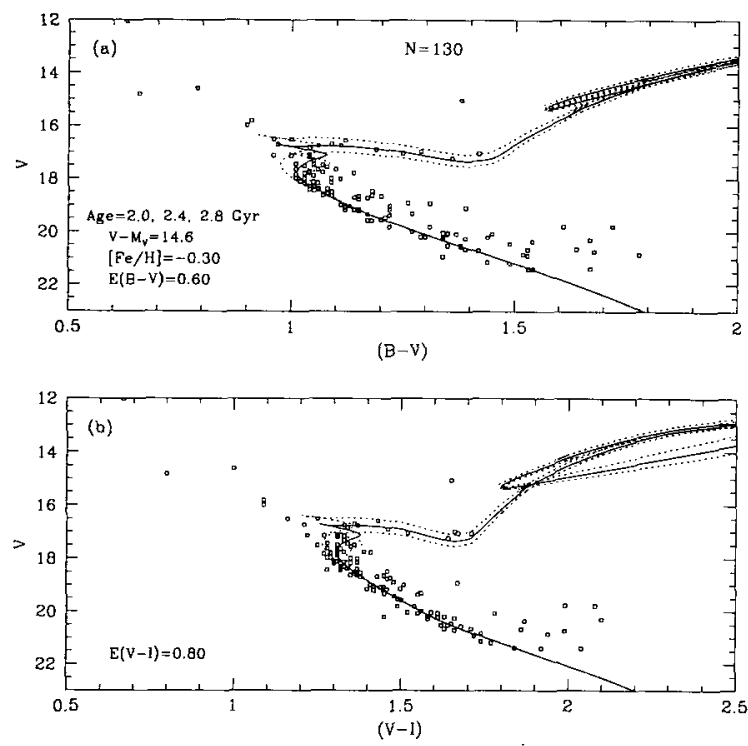

Fig. 5. - The Padova isochrone fitting on the $V-(B-V)$ (a) and $V-(V-I)($ b) color-magnitude diagrams with three age values of 2.0 (left dotted lines), 2.4 (solid lines), and 2.8 (right dotted lines) Gyr.
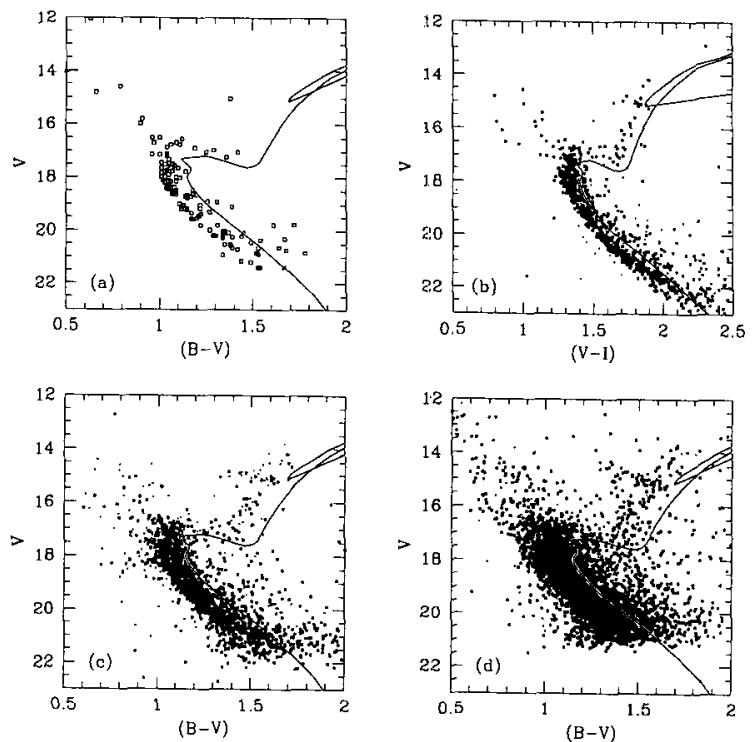

Fig. 6.- The Padova isochrone fitting using the parameters of Tr 5 derived by Kaluzny (1998) : age=4.1 Gyr, $[\mathrm{Fe} / \mathrm{H}]=0.00, E(B-V)=0.58, E(V-I)=0.765, A_{V}=$ $2.947 \times E(B-V)=1.71$, and $V-M_{V}=12.4+A_{V}=14.11$, on (a) the $V-(B-V)$ color-magnitude diagram for the stars commonly contained in the photometry files of the observing runs \#1 and \#2, (b) the $V-(V-I)$ colormagnitude diagram for the stars of the observing run \#1, (c) the $V-(B-V)$ color-magnitude diagram for the stars of the observing run $\# 2$, and (d) the $V-(B-V)$ colormagnitude diagram for the stars of the observing run \#3.

by comparing the colors of the RGCs of Tr 5 and M67 assuming the age of $4.1 \mathrm{Gyr}$ and metallicity the same as that of M67 for Tr 5 . He derived the distance of $\operatorname{Tr} 5$ as $(m-M)_{0} \approx 12.4$ by comparing the $I$-band luminosity of the RGC of $\operatorname{Tr} 5$ and the absolute $I$-band luminosity of red clump stars in the solar vicinity obtained from the Hipparcos distances $\left(M_{I}=-0.26\right)$.

In Figure 6 we showed the Padova isochrone fittings using the parameters of $\operatorname{Tr} 5$ derived by Kaluzny (1998) : age $=4.1 \mathrm{Gyr},[\mathrm{Fe} / \mathrm{H}]=0.00 \mathrm{dex}, E(B-V)=0.58$, $E(V-I)=0.765, A_{V}=2.947 \times E(B-V)=1.71$, and $V-M_{V}=12.4+A_{V}=14.11$, on (a) the $V-(B-$ $V)$ color-magnitude diagram for the stars commonly contained in the photometry files of observing runs \#1 and \#2, and color-magnitude diagrams for the stars of observing runs \#1, \#2, and \#3 in panels (b), (c), and (d), respectively. It is clearly seen that the parameters given by Kaluzny do not fit well the color-magnitude diagrams of observed stars.

Figure 7 shows the Padova isochrone fittings using the parameters of $\operatorname{Tr} 5$ derived in this study: age $=2.4$ $\mathrm{Gyr},[\mathrm{Fe} / \mathrm{H}]=-0.30 \mathrm{dex}, E(B-V)=0.60, E(V-I)=$ $0.80, A_{V}=3.26 \times E(B-V)=1.96$, and $V-M_{V}=$ $12.64+A_{V}=14.60$, on (a) the $V-(B-V)$ color- 

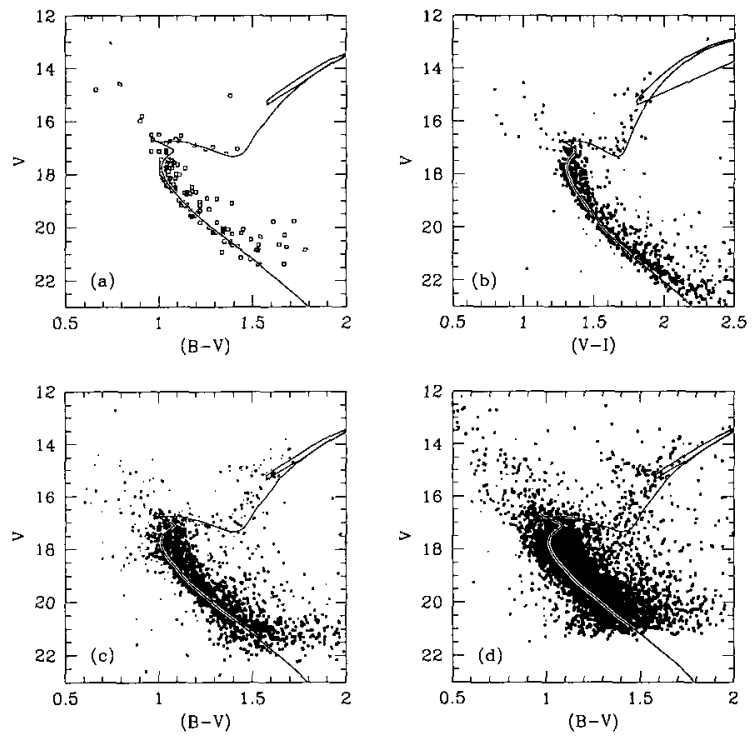

Fig. 7.- The Padova isochrone fitting using the parameters of $\operatorname{Tr} 5$ derived in this study : age $=2.4 \mathrm{Gyr}$, $[\mathrm{Fe} / \mathrm{H}]=-0.30, E(B-V)=0.60, E(V-I)=0.80, A_{V}=$ $3.26 \times E(B-V)=1.96$, and $V-M_{V}=12.64+A_{V}=14.60$, on (a) the $V-(B-V)$ color-magnitude diagram for the stars commonly contained in the photometry files of the observing runs \#1 and \#2, (b) the $V-(V-I)$ colormagnitude diagram for the stars of the observing run \#1, (c) the $V-(B-V)$ color-magnitude diagram for the stars of the observing run \#2, and (d) the $V-(B-V)$ colormagnitude diagram for the stars of the observing run \#3.

magnitude diagram for the stars commonly contained in the photometry files of the observing runs \#1 and \#2, and color-magnitude diagrams for the stars of the observing runs \#1, \#2, and \#3 in panels (b), (c), and (d), respectively. Comparison of Figures 6 and 7 shows that the parameters derived in this study better fits the observed color-magnitude diagrams. Since the ratio of field stars and binary stars to the member stars of $\mathrm{Tr}$ 5 might increases as we go from panels (a) to (d), the good fitting of the isochrone in Figure 7 (a) (and (b)) is noteworthy among the four panels.

\section{(b) $[\mathrm{Fe} / \mathrm{H}]-$-Galactocentric Radius Relation}

It is known that open clusters that are mainly in the Galactic disk follow the negative radial gradient of metallicity just as H II regions, bright blue stars, red giants, and planetary nebulae (Portinari \& Chiosi 1999; Hou, Prantzos, \& Boissier 2000). From the studies of open clusters, various authors have presented the slope of the Galactocentric radial $[\mathrm{Fe} / \mathrm{H}]$ gradient $\left(\Delta[\mathrm{Fe} / \mathrm{H}] / R_{g c}\right):-0.091 \pm 0.014 \mathrm{dex} \mathrm{kpc}^{-1}$ (Friel 1995), $-0.09 \mathrm{dex} \mathrm{kpc}-1$ (Carraro, $\mathrm{Ng}$, \& Portinari 1998), $-0.086 \pm 0.011$ dex $\mathrm{kpc}^{-1}$ (Park \& Lee 1999), and $-0.06 \pm 0.01 \mathrm{dex} \mathrm{kpc}^{-1}$ (Friel 1999). Based on updated

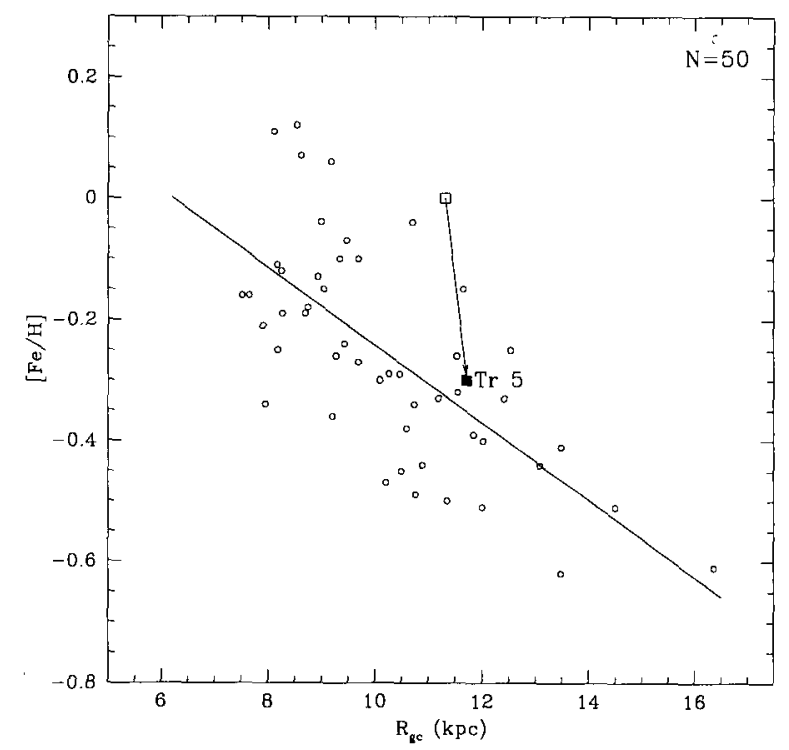

Fig. 8.- Radial abundance gradient for 50 old open clusters. The solid line is a least-squares fit to the data that yields an abundance gradient of $\Delta[\mathrm{Fe} / \mathrm{H}] / R_{g c}=-0.064 \pm$ $0.010 \mathrm{dex} \mathrm{kpc}^{-1}$. Open square and filled square are the position of Tr 5 based on the parameters given by Kaluzny (1998) and this study, respectively.

abundance calibration of spectroscopic indices measuring Fe and Fe-peak element blends on 39 old open clusters, Friel et al. (2002) have estimated the abundance gradient of $-0.06 \pm 0.01$ dex $\mathrm{kpc}^{-1}$ over a range in the Galactocentric radii of 7 to $16 \mathrm{kpc}$. From compilation of 119 open cluster catalogs, Chen, Hou, \& Wang (2003) have estimated the gradient of $-0.063 \pm 0.008$ dex $\mathrm{kpc}^{-1}$.

Compiling the data of old open clusters given by Friel (1995), Wee \& Lee (1996), Kassis, Friel, \& Phelps (1996), Lee (1997), Ann, Park, \& Kang (1998), and Friel et al. (2002), we made our catalog file of 50 old open clusters. We adopted the Galactocentric distance of the Sun as $8.5 \mathrm{kpc}$. Figure 8 shows the Galactocentric radial $[\mathrm{Fe} / \mathrm{H}]$ gradient for the 50 old open clusters. Open square and filled square are the position of $\mathrm{Tr}$ 5 based on the parameters given by Kaluzny (1998; $\left.R_{g c}=11.32 \mathrm{kpc}\right)$ and this study $\left(R_{g c}=11.71 \mathrm{kpc}\right)$, respectively. The solid line is a least-squares fit to the data that yields an $[\mathrm{Fe} / \mathrm{H}]$ gradient of $\Delta[\mathrm{Fe} / \mathrm{H}] / R_{g c}=$ $-0.064 \pm 0.010 \mathrm{dex} \mathrm{kpc}^{-1}$. This value is in good agreement with the values obtained by others, especially with the values derived from recent studies and the position of $\operatorname{Tr} 5$ in Figure 8 obtained by using the parameters derived in this study is more consistent with the mean trend of the other old open clusters. 
Table 1. Basic Information of Trumpler 5

\begin{tabular}{llc}
\hline \hline Parameter & Information & Reference \\
\hline Other names & C0634+094, OCL 494, Lund 237, Collinder 105 & Lyngå 1987 \\
$\alpha_{2000}, \delta_{2000}$ & $06^{h} 36^{m} 32^{s},+09^{\circ} \quad 29^{\prime} \quad 25^{\prime \prime}$ & This study \\
$l, b$ & $202 .^{\circ} 86,+01^{\circ} 05$ & Lyngå 1987 \\
Trumpler class & III $1 \mathrm{r}$ & Lynga 1987 \\
Reddening, $E(B-V)$ & $0.60 \pm 0.10 \mathrm{mag}$ & This study \\
Distance modulus, $V_{0}-M_{V}$ & $12.64 \pm 0.2 \mathrm{mag}$ & This study \\
Distance, d & $3.4 \pm 0.3 \mathrm{kpc}$ & This study \\
Galactocentric distance, $R_{g c}$ & $11.71 \pm 0.29 \mathrm{kpc}$ & This study \\
Metallicity, $[\mathrm{Fe} / \mathrm{H}]$ & $-0.30 \pm 0.10 \mathrm{dex}$ & This study \\
Age, $t$ & $2.4 \pm 0.2 \mathrm{Gyr}(\log t=9.38)$ & This study \\
\hline
\end{tabular}

\section{SUMMARY AND CONCLUSIONS}

We have presented the analysis of the photometry of the old open cluster Tr 5 using the CDS archival data obtained by Kaluzny. We have redetermined the reddening, distance, metallicity, and age of this cluster and summarized them in Table 1 . These parameters are more consistent with the Galactocentric radial $[\mathrm{Fe} / \mathrm{H}]$ gradient from other old open clusters.

SCK is grateful to In-Soo Yuk for providing his routines of the Padova isochrones. The authors would like to thank the referee, Prof. H. B. Ann for his careful reading of the manuscript which helped to clarify several issues. We employed catalogues from CDS/Simbad (Strasbourg) and Digitized Sky Survey images from the Space Telescope Science Institute. This work is the result of research activities (Astrophysical Research Center for the Structure and Evolution of the Cosmos ARCSEC) supported by Korea Science \& Engineering Foundation (HS).

\section{REFERENCES}

Ann, H. B., Park, Y.-H., \& Kang, Y.-W. 1998, "UBV CCD photometry of the open cluster Berkeley 2", JKAS, 31, 39

Bertelli, G., Bressan, A., Chiosi, C., Fagotto, F., \& Nasi, E. 1994, "Theoretical isochrones from models with new radiative opacities", A\&AS, 106, 275

Carraro, G., Ng, Y. K., \& Portinari, L. 1998, "On the Galactic disc age-metallicity relation", MNRAS, 296,1045

Chen, L., Hou, J. L., \& Wang, J. J. 2003, "On the Galactic disk metallicity distribution from open clusters. I. New catalogs and abundance gradient", AJ, 125, 1397

Dow, M. J., \& Hawarden, T. G. 1970, "The open cluster Trumpler 5", M. N. A. S. So. Africa, 29, 137

Friel, E. D. 1995, "The old open clusters of the Milky Way", ARAA, 33, 381
Friel, E. D. 1999, "Open clusters as a record of the past", Ap\&SS, 265, 271

Friel, E. D., Janes, K. A., Tavarez, M., Scott, J., Katsanis, R., Lotz, J., Hong, L., \& Miller, N. 2002, "Metallicities of old open clusters", AJ, 124, 2693

Guetter, H. H., \& Vrba, F. J. 1989, "Reddening and polarimetric studies toward IC 1805", AJ, 98, 611

Hou, J. L, Prantzos, N., \& Boissier, S. 2000, "Abundance gradients and their evolution in the Milky Way disk", A\&A, 362, 921

Janes, K., \& Adler, D. 1982, "Open clusters and Galactic structure", ApJS, 49, 425

Janes, K., \& Phelps, R. L. 1994, "The galactic system of old star clusters: The development of the galactic disk", AJ, 108, 1773

Kalinowski, J. K. 1974, "Observations of the carbon star V493 Monocerotis", IAU Info. Bull. on Var. Stars, No. 924

Kalinowski, J. K., Burkhead, M. S., \& Honeycutt, R. K. 1974, "On a possible carbon-star member of the old open cluster Trumpler 5", ApJ, 193, L77

Kalinowski, J. K. 1975, "The open cluster Trumpler 5", BAAS, 7, 542

Kalinowski, J. K. 1979, "The old disk cluster Trumpler 5", Ph.D. thesis, Indiana University

Kaluzny, J. 1994, "CCD photometry of distant open clusters. I. Berkeley 22, Berkeley 29 and Berkeley 54 ", A\&AS, 108, 151

Kaluzny, J. 1998, "CCD photometry of distant open clusters IV. Trumpler 5", A\&AS, 133, 25

Kassis, M., Friel, E. D., \& Phelps, R. L. 1996, "CCD photometry of the old open cluster 092-SC18", AJ, 111,820

Lee, M. G. 1997, "UBVI CCD photometry of the remote old open cluster AM-2", AJ, 113, 729

Lyngå, G. 1987, Catalogue of Open Cluster Data (Observatoire de Strassbourg, Centre de Données Stellaires) 
Montgomery, K. A., Marschall, L. A., \& Janes, K. A. 1993, "CCD photometry of the old open cluster M67", AJ, 106, 181

Park, H. S., \& Lee, M. G. 1999, "UBVI charge-coupled device photometry of two old open clusters NGC 1798 and 2192", MNRAS, 304, 883

Phelps, R. L., Janes, K. A., \& Montgomery, K. A. 1994, "Development of the Galactic disk: A search for the oldest open clusters", AJ, 107, 1079

Piccirillo, J., Kalinowski, J. K., \& Wing, R. F. 1977, "The red giants of Trumpler 5", BAAS, 9, 637

Portinari, L., \& Chiosi, C. 1999, "On star formation and chemical evolution in the Galactic disk", A\&A, 350,827

Sung, H. 2001, "Young open clusters and early stellar evolution", Star Clusters, Korea Astronomy Observatory Workshop, eds. S.-L. Kim and J.-M. Kyeong, 1 (in Korean)

Sung, H., \& Bessell, M. S. 1999, "UBVI CCD photometry of M35 (NGC 2168)", MNRAS, 306, 361

Wee, S.-O., \& Lee, M. G. 1996, "Washington CCD photometry of the old open cluster NGC 1245", JKAS, 29,181 